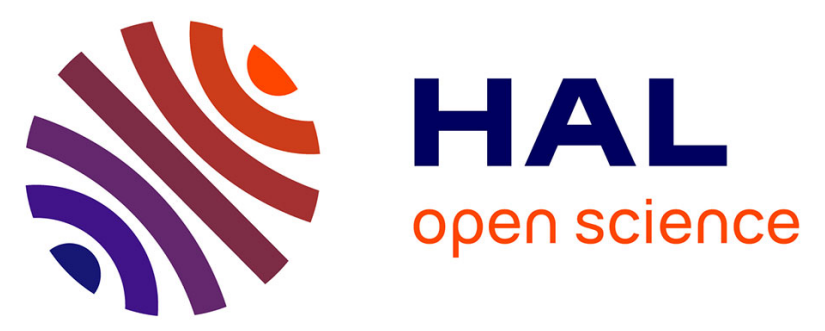

\title{
Coreference Resolution for French Oral Data: Machine Learning Experiments with ANCOR
}

Adèle Désoyer, Frédéric Landragin, Isabelle Tellier, Anaïs Lefeuvre, Jean-Yves Antoine, Marco Dinarelli

\section{- To cite this version:}

Adèle Désoyer, Frédéric Landragin, Isabelle Tellier, Anaïs Lefeuvre, Jean-Yves Antoine, et al.. Coreference Resolution for French Oral Data: Machine Learning Experiments with ANCOR. 17th International Conference on Intelligent Text Processing and Computational Linguistics (CICLing'2016), Apr 2016, Konya, Turkey. hal-01344977

\section{HAL Id: hal-01344977 \\ https://hal.science/hal-01344977}

Submitted on 13 Jul 2016

HAL is a multi-disciplinary open access archive for the deposit and dissemination of scientific research documents, whether they are published or not. The documents may come from teaching and research institutions in France or abroad, or from public or private research centers.
L'archive ouverte pluridisciplinaire HAL, est destinée au dépôt et à la diffusion de documents scientifiques de niveau recherche, publiés ou non, émanant des établissements d'enseignement et de recherche français ou étrangers, des laboratoires publics ou privés. 


\title{
Coreference Resolution for French Oral Data: Machine Learning Experiments with ANCOR
}

\author{
Adèle Désoyer ${ }^{1,2}$, Frédéric Landragin ${ }^{1}$, Isabelle Tellier ${ }^{1}$, Anaïs Lefeuvre ${ }^{3}$, \\ Jean-Yves Antoine ${ }^{3}$, and Marco Dinarelli ${ }^{1}$ \\ 1 Lattice, CNRS, ENS, Université de Paris 3, Université Sorbonne Paris Cité, \\ PSL Research University, Paris, France \\ \{frederic.landragin, isabelle.tellier, marco.dinarelli\}@ens.fr \\ 2 Modyco, CNRS, Université Paris Ouest - Nanterre La Défense, France \\ adele.desoyer@gmail.com \\ 3 LI, CNRS, Université François Rabelais de Tours, France \\ \{anais.lefeuvre, jean-yves.antoine\}@univ-tours.fr
}

\begin{abstract}
We present CROC (Coreference Resolution for Oral Corpus), the first machine learning system for coreference resolution in French. One specific aspect of the system is that it has been trained on data that come exclusively from transcribed speech, namely ANCOR (ANaphora and Coreference in ORal corpus), the first large-scale French corpus with anaphorical relation annotations. In its current state, the CROC system requires pre-annotated mentions. We detail the features used for the learning algorithms, and we present a set of experiments with these features. The scores we obtain are close to those of state-of-the-art systems for written English.
\end{abstract}

Keywords: mention-pair model, dialogue corpus, coreference resolution, machine learning

\section{Introduction}

Coreference Resolution has now become a classical task in NLP. This task consists in identifying coreference chains of mentions in texts. Supervised machine learning approaches are now largely dominant in this domain, but they require annotated corpora. No such corpus was available for French so far. In this paper, we first describe ANCOR, the first large-scale French corpus annotated with coreferent mentions. It is made of transcribed oral data, which is a specificity relatively to corpora available for other languages. We then present CROC, a baseline system which has been learned with ANCOR, and a variant. Their performances are very close to those observed on coreference resolution challenges for English.

\section{The ANCOR Corpus}

We present ANCOR, a French corpus annotated with coreference relations which is freely available and large enough to serve the needs of data-driven approaches 
in NLP. With a total of 488,000 lexical units, ANCOR is among the largest coreference annotated corpora available at present and the only one of comparable size in French.

The main originality of this resource lies in the focus on spoken language. Nowaday systems using NLP for Information retrieval or extraction, for text summarization or even for machine translation have mainly been designed for written language. Oral language presents some interesting specificities, such as the absence of sentence units, the lack of punctuation, the presence of speech disfluencies, and, obviously, the grammatical variability of utterances. See [26] for a more detailed list of oral specific features which make French oral processing a big challenge.

\subsection{Presentation of the corpus}

The ANCOR corpus is made of spoken French and it aims at representing a certain variety of spoken types. It integrates three different corpora that were already transcribed during previous research projects (Table 1). The first and larger one has been extracted from the ESLO corpus, which collects sociolinguistic interviews [16]. This corpus can be divided in two sub-corpora (ESLOANCOR and ESLO-CO2), corresponding to two distinct periods of recordings. It is characterized by a low level of interactivity. On the opposite, OTG and Accueil_UBS concern highly interactive Human-Human dialogues [18]. These two corpora differ by the media of interaction: direct conversation for the first one, phone call for the other one. Conversational speech (OTG and Accueil) only represents $7 \%$ of the total because of the scarcity of such free resources in French. All corpora are freely distributed under a Creative Commons license.

\begin{tabular}{|c|c|c|c|c|}
\hline Corpus & Speech type & Interactivity & Size & Duration \\
\hline $\begin{array}{l}\mathrm{ESLO} \\
\mathrm{ESLO} \mathrm{A}_{-} \mathrm{ANCOR} \\
\text { ESLO_CO2 } \\
\end{array}$ & interview & low & $\begin{array}{r}452,000 \text { words } \\
417,000 \text { words } \\
35,000 \text { words }\end{array}$ & $\begin{array}{r}27,5 \text { hours } \\
25 \text { hours } \\
2.5 \text { hours } \\
\end{array}$ \\
\hline OTG & $\begin{array}{c}\text { task oriented conv- } \\
\text { ersational speech }\end{array}$ & high & 26,000 words & 2 hours \\
\hline Accueil_UBS & $\begin{array}{l}\text { phone conversa- } \\
\text { tional speech }\end{array}$ & high & 10,000 words & 1 hour \\
\hline
\end{tabular}

Table 1. ANCOR source corpus types and characterization

\subsection{Annotation scheme}

The corpus has been fully annotated by hand on the Glozz platform [15]. Glozz produces a stand-off XML file structured according to a DTD that was specifically designed for ANCOR (it has also been translated in the MMAX2 format 
for portability purposes). This stand-off annotation allows a multi-layer work and enrichments through time.

The scope of annotation takes into account all noun phrases (NP from now) including pronouns but strictly restricts to them. As a result, the annotation scheme discards coreferences involving verbal or propositional mentions which have been annotated in the OntoNotes corpus. This restriction was mainly intended to favor data reliability by focusing on easily identifiable mentions [12].

Another specificity of the scheme is the annotation of isolated mentions. NPs are annotated even if they are not involved in any anaphoric relation, and this is a real added value for coreference resolution since the detection of singletons is known to be a difficult task [20].

We followed a detailed annotation scheme in order to provide useful data for deep linguistic studies and machine learning. Every nominal group is thus associated with the following features:

- Gender,

- Number,

- Part of Speech (only mentions have been annotated with these features, the corpus does not provide any morpho-syntactic annotation level on its own for other tokens),

- Definition (indefinite, definite, demonstrative or expletive form),

- PP: inclusion or not in a prepositional phrase,

- NE: Named Entity Type, as defined in the Ester2 coding scheme [8],

- NEW: discourse new vs. subsequent mention.

Coders were asked to link subsequent mentions with the first mention of the corresponding entity (discourse new) and to classify the relation among five different types of coreference or anaphora:

- Direct coreference: coreferent mentions are NP with the same lexical head.

- Indirect coreference: NP coreferent mentions with distinct lexical head (schooner ... vessel).

- Pronominal anaphora: the subsequent coreferent mention is a pronoun.

- Bridging anaphora: non coreference, but the subsequent mention depends on its antecedent for its referential interpretation (meronomy for instance: the schooner ... its bowsprit).

- Bridging pronominal anaphora: the subsequent mention is a pronoun. Its interpretation depends on its antecedent but the two mentions are not coreferent (for instance: the hostel ... they are welcoming).

This annotation scheme is quite similar to previous works on written language $[5,28]$. Since ANCOR represents the first large coreference corpus available for French, it is important that the resource should concern researchers that are working on written documents too. Unlike [9], we did not distinguish between several sub-categories of bridging anaphora. We consider such a refined taxonomy to exceed the present needs of NLP while introducing a higher subjectivity in the annotation process. For the same reasons, we did not consider the relation of near-identity proposed in [20]. Recent experiments have shown that near-identity leads to a rather low inter-coders agreement [3]. 


\subsection{Distributional data}

This section gives a general outline of the annotated data, to roughly show what should be found in the resource.

Table 2 details how the mentions and relations are distributed among the subcorpora. With more than 50,000 relations and 100,000 mentions, ANCOR should fulfill the needs of representativity for linguistic studies and machine learning experiments. Table 3 shows that the repartition of nominal and pronominal entities is noticeable stable among the four corpora and leads to a very balanced overall distribution ( $51.2 \%$ vs. $48.8 \%)$.

\begin{tabular}{|l|r|r|}
\hline Corpus & Number of mentions & Number of relations \\
\hline ESLO & 106,737 & 48,110 \\
$E S L O \_A N C O R$ & 97,939 & 44,597 \\
ESLO_CO2 & 8,798 & 3,513 \\
\hline OTG & 7,462 & 2,572 \\
\hline Accueil_UBS & 1,872 & 655 \\
\hline Total & 116,071 & 51,337 \\
\hline
\end{tabular}

Table 2. Content of the different sub-corpora

\begin{tabular}{|l|r|r|r|}
\hline Entities & Nominal & Pronouns & \% of NE \\
\hline ESLO_ANCOR & 51.8 & 48.4 & 66.3 \\
ESLO_CO2 & 49.4 & 50.6 & 52,4 \\
OTG & 47.5 & 52.5 & 48.6 \\
Accueil_UBS & 48.5 & 51.5 & 43.3 \\
\hline Total & 51.2 & 48.8 & 59.8 \\
\hline
\end{tabular}

Table 3. Mentions: distributional information

This observation certainly results from a general behavior of French speakers: pronominal anaphora are indeed an easy way for them to avoid systematic repetitions in a coreference chain.

In addition, ANCOR contains around 45,000 annotated Named Entities (NE). Therefore, it should stand for a valuable resource for NE recognition applications. 26,722 NE have been annotated as persons, 3,815 as locations, 1,746 as organizations, 1,496 as amounts, 1,390 for time mentions and 1,185 as products.

Finally, Table 5 presents the distribution of coreference/anaphora relations. Once again, strong regularities between the sub-corpora are observed. In particular, direct coreference and pronominal anaphora are always prevalent. AN- 


\begin{tabular}{|c|c|c|c|c|c|}
\hline Person & Location & Organization & Amount & Time & Product \\
\hline 26,722 & 3,815 & 1,746 & 1,496 & 1,390 & 1,185 \\
\hline
\end{tabular}

Table 4. Most frequent named entities in ANCOR

\begin{tabular}{|c|c|c|c|c|}
\hline Direct & Indirect & Pronominal & Bridging & Bridging pronominal \\
\hline 38,2 & 6,7 & 41,1 & 9,8 & 1,0 \\
\hline
\end{tabular}

Table 5. Relations: distributional percentages

COR contains around 20,000 occurrences of direct coreference and pronominal anaphora which are always prevalent through the corpus.

\subsection{Annotation reliability estimation}

The estimation of data reliability is still an open issue on coreference annotation. Indeed, the potential discrepancies between coders frequently lead to alignment mismatches that prevent the direct application of standard reliability measures $[19,1,15]$. We propose to overcome this problem by assessing separately the reliability of 1) the delimitation of the relations and 2) the annotation of their types. More precisely, three experiments have been conducted:

1. Firstly, we have asked 10 experts to delimitate the relations on an extract of ANCOR. These coders were previously trained on the annotation guide. We computed, on the basis of every potential pair of mentions, standard agreement measures: $\kappa$ [4], $\alpha$ [11] and $\pi$ [22]. This experiment aims above all at evaluating the degree of subjectivity of the task rather than the reliability of the annotated data, since the experts were not the coders of the corpus.

2. On the contrary, the second experiment concerned the annotators and the supervisor of the corpus. We asked them to re-annotate an extract of the corpus. Then we computed intra-coders agreement through a comparison to what they really performed on the actual corpus. This experiment aims at providing an estimation of the coherence of data.

\begin{tabular}{|l|r|r|r|r|r|}
\hline Corpus & ESLO_Ancor & ESLO_CO2 & OTG & Accueil_UBS & Total \\
\hline Direct & 41,1 & 35,2 & 39,7 & 40,5 & 38,2 \\
\hline Indirect & 7,3 & 11,2 & 6,1 & 7,5 & 6,7 \\
\hline Pronoun anaphora & 43,9 & 38,2 & 46,4 & 46,0 & 41,1 \\
\hline Bridging anaphora & 10,4 & 14,4 & 13,5 & 11,0 & 9,8 \\
\hline Pronoun bridging & 0,9 & 1,0 & 3,3 & 0,6 & 1,0 \\
\hline
\end{tabular}

Table 6. Coreference/anaphora: distributional percentages 
3. Finally, we asked our 10 first experts to attribute one type to a selection of relations that were previously delimited in the ANCOR corpus. We then computed agreement measures on the resulting type annotation.

\begin{tabular}{|l|r|r|r|}
\hline Agreement & $\kappa$ & $\pi$ & $\alpha$ \\
\hline Delimitation: inter-coder agreement & 0.45 & 0.45 & 0.45 \\
\hline Delimitation: intra-coder agreement & 0.91 & 0.91 & 0.91 \\
\hline Type categorization: inter-coder agreement & 0.80 & 0.80 & 0.80 \\
\hline
\end{tabular}

Table 7. Agreement measures for the ANCOR corpus

We observe on table 7 very close results with the three considered reliability metrics (no difference before the 4th decimal). This is not surprising since we consider a binary distance between classes. The inter-coder agreement on delimitation is rather low $(0.45)$. One should however note that this measure should be biased by our discourse-new coding scheme. Indeed, if a disagreement only concerns the first mention of a coreference chain, all the subsequent relations will unjustifiably penalize the reliability estimation. Further measures to come with the chain coding scheme will soon give an estimation of this potential bias. Anyway, this rather low agreement suggests that the delimitation task is highly prone to subjectivity, even when coders are trained. In particular, a detailed analysis of confusion matrices shows that most discrepancies occur between the delimitation of a bridging anaphora and the decision to not annotate a relation. Besides, this kind of disagreement appears to be related to personal idiosyncrasies. On the contrary, the results become very satisfactory when you consider intra-coders agreement (0.91). This means that our coders followed a very coherent strategy of annotation, under the control of the supervisor. This coherence is, in our opinion, an essential guarantee of reliability. Lastly we observed very good agreements on the categorization task (0.80), which reinforce our decision not to consider near-identity or detailed bridging types.

\section{Machine Learning for Coreference Resolution}

Coreference Resolution has become a classical task for NLP challenges, e.g. those organized by MUC (http://www.itl.nist.gov/iaui/894.02/related_ projects/muc/proceedings/muc_7_toc.html), ACE (http://www.itl.nist. gov/iad/mig//tests/ace/), SemEval (http://semeval2.fbk.eu/semeval2. php?location=tasks) or CoNLL (http://conll.cemantix.org/2011/ \& then http://conll.cemantix.org/2012/). But none of these challenges included French corpora. For French, as no labelled data were available before ANCOR, only hand-crafted systems have been proposed so far $[27,13]$. We rely instead on machine learning approaches. In this section we present our system, named 
CROC for "Coreference Resolution for Oral Corpus". It only treats the coreference task. We thus suppose that every mention has already been recognized and associated with its specific features (see section 2.2). The system was trained on the ANCOR_Centre corpus, using the WEKA machine learning platform [29].

\subsection{Brief state of the art}

Several approaches have been proposed to reformulate coreference resolution as a machine leaning problem. The first and simpler one is the pairwise approach which proposes to classify every possible pair of referring mentions as co-referential or not. This approach assumes that referring mentions are provided (as we do in this paper) and requires a post-processing to build global chains from a set of local pairs. In order to do so, $[23,17]$ apply a Closest-First strategy, which attaches a mention to its closest (on the left) co-referring other mention, whereas $[2,24]$ propose a Best-First strategy, taking into account "coreferential probabilities".

Twin-candidate models [31] are variants of the pairwise approach in which the classification is applied to triples instead of pairs: an anaphoric mention and two candidates for its antecedent (the result being either first or second depending on which of the two candidates is the selected antecedent). criteria between candidates. Other more sophisticated models such as the Twin-candidate [31], mention-ranking [6] or entity-mention [30] have also been proposed. Our coreference resolution system is a baseline, it will thus use the pairwise and Closest-First strategies.

\subsection{Representation of the data in CROC}

\begin{tabular}{|l|l|l|l|l|l|l|}
\hline System & Language & Corpus & MUC & B3 & CEAF & BLANC \\
\hline$[23]$ & English & MUC-7 & 60.4 & - & - & - \\
{$[17]$} & English & MUC-7 & 63.4 & - & - & - \\
{$[25]$} & English & ACE-2003 & 67.9 & 65.9 & - & - \\
{$[24]$} & English & MUC-7 & 62.8 & 79.4 & - & - \\
{$[10]$} & English & ACE-2004 & 67.0 & 77.0 & - & - \\
{$[12]$} & English & CoNNL-2012 & 68.8 & 54.56 & 50.20 & - \\
{$[13]$} & French & heterogeneous & 36 & 69.7 & 55 & 59.5 \\
\hline
\end{tabular}

Table 8. Results of end-to-end systems

We have developed CROC as a baseline system which follows the pairwise and closest-first strategies. Pairwise systems rely on a good representation of pairs of mentions. In state of the art models, this representation is usually based on the classical set of features proposed in [23], augmented by those of [17]. For 


\begin{tabular}{|l|l|l|l|l|l|l|}
\hline System & Language & Corpus & MUC & B3 & CEAF & BLANC \\
\hline$[31]$ & English & MUC-7 & 60.2 & - & - & - \\
{$[14]$} & English & ACE-2 & 80.7 & 77.0 & 73.2 & 77.2 \\
{$[7]$} & English & ACE-2 & 71.6 & 72.7 & 67.0 & - \\
{$[2]$} & English & ACE-2004 & 75.1 & 80.8 & 75.0 & 75.6 \\
\hline CROC & French & ANCOR & $\mathbf{6 3 . 4 5}$ & $\mathbf{8 3 . 7 6}$ & $\mathbf{7 9 . 1 4}$ & $\mathbf{6 7 . 4 3}$ \\
One-Class SVM & French & ANCOR & $\mathbf{6 1 . 7 3}$ & $\mathbf{8 4 . 5 8}$ & $\mathbf{8 0 . 4 1}$ & $\mathbf{6 9 . 6 6}$ \\
\hline
\end{tabular}

Table 9. Results of systems starting with pre-annotated mentions

our experiments, we used all of these features when they are available in the corpus, plus some new ones we designed. The added features concern speakers and speech turns: they are specific to oral data (in particular to dialogues). One of our purposes is to evaluate the impact of these oral-specific features on the results. For each candidate pair of mentions (i,j), our set of features includes (cf. also table 10):

1. features characterizing each mention $\mathrm{i}$ and $\mathrm{j}$ :

- at the morphological level: is it a pronoun? is it a definitive SN? is it a demonstrative SN?

- at the enunciative level: is it a new mention?

- at the semantic level: is it a named entity? of which type? Note that no freely available reliable semantic network is available for French, so no other semantic feature was used.

2. relational features, characterizing the pair:

- at the lexical level: are the mentions strictly equal? partly equal?

- at the morphosyntactic level: do they agree in gender? in number? Note that, in French, even if personal pronouns like "il" (he), "elle" (she)... agree in gender and number with their antecedent, possessive pronouns like "son", "sa"... (his, her...) agree with the noun they introduce and not with the referred antecedent.

- at the spatial level: how many characters/tokens/mentions/speech turns separate them?

- at the syntactic level: is one of the mentions included in the other one?

- at the contextual level: are their preceding/next tokens the same?

- at the enunciative level: are they produced by the same speaker?

\subsection{Baseline results}

From the initial corpus, we kept $60 \%$ of data for learning, $20 \%$ for development, and $20 \%$ for test. In order to estimate the influence of the learning corpus size, we distinguished three sets: a small one (71,881 instances), a medium one $(101,919$ instances) and a big one (142,498 instances). In these sets, $20 \%$ of instances are 


\begin{tabular}{|c|c|c|c|}
\hline & Features & Definitions & Possible values \\
\hline 1 & $m_{1-T Y P E}$ & syntactic category of $m_{1}$ & $\{\mathrm{~N}, \mathrm{PR}, \mathrm{UNK}, \mathrm{NULL}\}$ \\
\hline 2 & $m_{2-\mathrm{TYPE}}$ & syntactic category of $m_{2}$ & $\{\mathrm{~N}, \mathrm{PR}, \mathrm{UNK}, \mathrm{NULL}\}$ \\
\hline 3 & $m_{1 \_\mathrm{DEF}}$ & definition of $m_{1}$ & \multirow{2}{*}{$\begin{array}{l}\{\text { UNK, INDEF, EXPL, } \\
\text { DEF_SPLE, DEF_DEM }\}\end{array}$} \\
\hline 4 & $m_{2 \_ \text {DEF }}$ & definition of $m_{2}$ & \\
\hline 5 & $m_{1 \_ \text {GENDER }}$ & gender of $m_{1}$ & $\{\mathrm{M}, \mathrm{F}, \mathrm{UNK}, \mathrm{NULL}\}$ \\
\hline 6 & $m_{2}$ GENDER & gender of $m_{2}$ & $\{\mathrm{M}, \mathrm{F}, \mathrm{UNK}, \mathrm{NULL}\}$ \\
\hline 7 & $m_{1 \_ \text {NUMBER }}$ & number of $m_{1}$ & $\{\mathrm{SG}, \mathrm{PL}, \mathrm{UNK}, \mathrm{NULL}\}$ \\
\hline 8 & $m_{2 \_ \text {NUMBER }}$ & number of $m_{2}$ & $\{\mathrm{SG}, \mathrm{PL}, \mathrm{UNK}, \mathrm{NULL}\}$ \\
\hline 9 & "m $m_{1 \_N E W}$ & is a new entity introduced by $m_{1}$ ? & $\{$ YES, NO, UNK, NULL $\}$ \\
\hline 10 & $m_{2 \_\mathrm{NEW}}$ & is a new entity introduced by $m_{2}$ ? & $\{$ YES, NO, UNK, NULL $\}$ \\
\hline 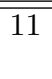 & $m_{1 \_\mathrm{EN}}$ & entity type of $m_{1}$ & \multirow{2}{*}{$\begin{array}{l}\text { \{PERS, FONC, LOC } \\
\text { ORG, PROD, TIME, NO, } \\
\text { AMOUNT, UNK, NULL } \\
\text { EVENT }\}\end{array}$} \\
\hline 12 & $m_{2 \_ \text {EN }}$ & entity type of $m_{2}$ & \\
\hline 13 & ID_FORM & are $m_{1}$ and $m_{2}$ forms identical? & $\{$ YES, NO, NA $\}$ \\
\hline 14 & ID_SUBFORM & are there identical sub-forms? & $\{\mathrm{YES}, \mathrm{NO}, \mathrm{NA}\}$ \\
\hline 15 & INCL_RATE & tokens covering ratio & REAL \\
\hline 16 & COM_RATE & common tokens ratio & REAL \\
\hline 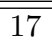 & $\begin{array}{l}\text { ID_DEF } \\
\end{array}$ & is there definition equality? & $\{$ YES, NO, NA $\}$ \\
\hline 18 & ID_TYPE & is there type equality? & $\{$ YES, NO, NA $\}$ \\
\hline 19 & ID_EN & is there named entity type equality? & $\{\mathrm{YES}, \mathrm{NO}, \mathrm{NA}\}$ \\
\hline 20 & ID_GENDER & is there gender equality? & $\{$ YES, NO, NA $\}$ \\
\hline 21 & ID_NUMBER & is there number equality? & $\{\mathrm{YES}, \mathrm{NO}, \mathrm{NA}\}$ \\
\hline 22 & DISTANCE_MENTION & "distance (number of mentions) & REAL \\
\hline 23 & DISTANCE_TURN & distance (number of speech turns) & REAL \\
\hline 24 & DISTANCE_WORD & distance (number of words) & REAL \\
\hline 25 & DISTANCE_CHAR & distance (number of characters) & REAL \\
\hline 26 & EMBEDDED & embedding of $m_{2}$ in $m_{1}$ ? & $\mid\{\mathrm{YES}, \mathrm{NO}, \mathrm{NA}\}$ \\
\hline 27 & ID_PREVIOUS & are previous tokens identical? & $\{\mathrm{YES}, \mathrm{NO}, \mathrm{NA}\}$ \\
\hline 28 & ID_NEXT & are next tokens identical? & $\{\mathrm{YES}, \mathrm{NO}, \mathrm{NA}\}$ \\
\hline 29 & ID_SPK & are speakers identical? & $\{\mathrm{YES}, \mathrm{NO}, \mathrm{NA}\}$ \\
\hline 30 & ID_NEW & are discursive status identical? & $\{$ YES, NO, NA $\}$ \\
\hline
\end{tabular}

Table 10. CROC complete feature set 
coreferent pairs that are directly extracted from the corpus, and $80 \%$ are notcoreferent pairs (negative examples). We also tested different sets of features. In particular, we distinguished three sets: a first one that includes all features, a second one with only relational features, and a third one with all the features that are not linked to oral specificities. A last source of variation concerned the machine learning algorithm used: we tried decision trees, SVM (SMO with default parameters), and Naive Bayes using the WEKA platform.

Experiments involving development data showed that the best-performing model is the one calculated by SVM on small training set of data described by all features. Test data are submitted to this model, and the results are filtered by the Closest-First method, retaining only the closest antecedent if several pairs involving a mention were found coreferent. We present in tables 8 and 9 the results of some state-of-the-art coreference resolution systems, for the four metrics dedicated to coreference resolution. Oral-specific features do not significantly improve the results.

\subsection{One-class SVM}

One of the main problems when using the pairwise approach is that, in order to train the binary classification model, artificial negative instances must be generated. Since there is no information to decide whether a pair of not-coreferent mentions is plausible or not, all possible pairs must be generated. The number of such pairs is polynomial in the length of a given mention set in a text, and this in turn means that negative instances are by far more numerous than positive instances. Since this may create a problem of unbalanced representation of positive and negative classes in SVM, heuristics have been proposed to filter out part of negative instances $[23,17]$. Despite such heuristics, negative instances are still much more than positive ones.

In order to overcome this problem we investigated the use of models which do not need negative instances. One such model still belongs to the SVM family, namely One-class SVM [21]. One-class SVM only needs positive instances, and instead of separating positive and negative instances from each other, separates positive instances from the origin. In order to make a comparison with our baseline, we trained such a model with exactly the same data and features. The results are shown in table 9, line One-Class SVM. Our research in this direction is in progress, but we can see that currently results obtained with this approach are roughly equivalent to baseline results.

\section{Conclusion and Perspective}

Most current researches on coreference resolution concern written language. In this paper, we presented experiments that were conducted on ANCOR, a large French corpus based on speech transcripts, annotated with rich information and coreference chains. This corpus represents the first significant effort to provide sufficient coreference training data in French for machine learning approaches. 
We described CROC, a baseline approach for automatic coreference resolution on French, as well as another machine learning approach based on one-class SVM. Our first results are roughly equivalent to state-of-the-art performances, which suggests that standard ML approaches for coreference resolution should apply satisfactory on spoken language.

For further investigation, we plan to more carefully study the impact of the various corpus origins on the final results. Does the speech type and/or the level of interactivity influence the way co-reference chains are built in dialogues? To better compare our results with the state of the art, other more complex learning models also need to be tested on these data. And finally, to provide a real endto-end system, we have to automatically identity the mentions and their specific features, as a pre-processing step.

Acknowledgments. This work was supported by grant ANR-15-CE38-0008 ("DEMOCRAT" project) from the French National Research Agency (ANR), and by APR Centre-Val-de-Loire region ("ANCOR" project).

\section{References}

1. Artstein, R., Poesio, M.: Inter-Coder Agreement for Computational Linguistics. Computational Linguistics 34(4), 555-596 (Dec 2008)

2. Bengtson, E., Roth, D.: Understanding the Value of Features for Coreference Resolution. In: Proceedings of EMNLP 2010. pp. 236-243 (2008)

3. Broda, B., Niton, B., Gruszczynski, W., Ogrodniczuk, M.: Measuring readability of polish texts: Baseline experiments. In: Proceedings of the Ninth International Conference on Language Resources and Evaluation. Reykjavik, Iceland (2014)

4. Cohen, J.: A Coefficient of Agreement for Nominal Scales. Educational and Psychological Measurement 20, 37-46 (1960)

5. van Deemter, K., Kibble, R.: On coreferring: Coreference in muc and related annotation schemes. Computational Linguistics 26(4), 629-637 (2000)

6. Denis, P.: New Learning Models for Robust Reference Resolution. Ph.D. thesis, University of Texas at Austin (2007)

7. Denis, P., Baldridge, J.: Specialized models and ranking for coreference resolution. In: Proceedings of the Conference on Empirical Methods in Natural Language Processing. pp. 660-669. EMNLP'08 (2008)

8. Galliano, S., Gravier, G., Chaubard, L.: The ester2 evaluation campaign for the rich transcription of french radio broadcasts. In: Proceedings of Interspeech (2009)

9. Gardent, C., Manuélian, H.: Création d'un corpus annoté pour le traitement des descriptions définies. TAL 46(1), 115-139 (2005)

10. Haghighi, A., Klein, D.: Coreference resolution in a modular, entity-centered model. In: Human Language Technologies: The 2010 Annual Conference of the North American Chapter of the Association for Computational Linguistics. pp. 385-393 (2010)

11. Krippendorff, K.: Content Analysis: an Introduction to its Methodology. SAGE Publications, Inc. (2004)

12. Lassalle, E.: Structured Learning with Latent Trees: A Joint Approach to Coreference Resolution. Ph.D. thesis, Université Paris Diderot (2015) 
13. Longo, L.: Vers des moteurs de recherche intelligents : un outil de détection automatique de thèmes. Ph.D. thesis, Université de Strasbourg (2013)

14. Luo, X., Ittycheriah, A., Jing, H., Kambhatla, N., Roukos, S.: A mentionsynchronous coreference resolution algorithm based on the bell tree. In: Proceedings of the 42nd Annual Meeting on Association for Computational Linguistics (2004)

15. Mathet, Y., Widlöcher, A.: Une approche holiste et unifiée de l'alignement et de la mesure d'accord inter-annotateurs. In: Actes de TALN. pp. 1-12. ATALA (2011)

16. Muzerelle, J., Lefeuvre, A., Schang, E., Antoine, J.Y., Pelletier, A., Maurel, D., Eshkol, I., Villaneau, J.: Ancor_centre, a large free spoken french coreference corpus: description of the resource and reliability measures. In: Proceedings of the Ninth International Conference on Language Resources and Evaluation. Reykjavik, Iceland (2014)

17. Ng, V., Cardie, C.: Improving Machine Learning Approcahes to Corefrence Resolution. In: Proceedings of ACL'02. pp. 104-111 (2002)

18. Nicolas, P., Letellier-Zarshenas, S., Schadle, I., Antoine, J.Y., Caelen, J.: Towards a large corpus of spoken dialogue in french that will be freely available: the "parole publique" project and its first realisations. In: Proceedings of LREC (2002)

19. Passonneau, R.J.: Computing reliability for coreference annotation. In: Proceedings of LREC. pp. 1503-1506 (2004)

20. Recasens, M.: Coreference : Theory, Resolution, Annotation and Evaluation. Ph.D. thesis, University of Barcelona (2010)

21. Schölkopf, B., Platt, J.C., Shawe-Taylor, J.C., Smola, A.J., Williamson, R.C.: Estimating the support of a high-dimensional distribution. Neural Comput. 13(7), 1443-1471 (2001)

22. Scott, W.: Reliability of content analysis: The case of nominal scale coding. Public Opinions Quaterly 19, 321-325 (1955)

23. Soon, W.M., Ng, H.T., Lim, D.C.Y.: A Machine Learning Approach to Coreference Resolution of Noun Phrases. Computational Linguistics 27(4), 521-544 (2001)

24. Stoyanov, V., Cardie, C., Gilbert, N., Riloff, E., Buttler, D., Hysom, D.: Reconcile : A Coreference Resolution Research Platform. Tech. rep., Cornell University (2010)

25. Stoyanov, V., Gilbert, N., Cardie, C., Riloff, E.: Conundrums in noun phrase coreference resolution: Making sense of the state-of-the-art. In: Proceedings of the Joint Conference of the 47th Annual Meeting of the ACL and the 4th International Joint Conference on Natural Language Processing. pp. 656-664 (2009)

26. Tellier, I., Eshkol, I., Taalab, S., Prost, J.P.: Pos-tagging for oral texts with crf and category decomposition. Research in Computing Science 46, 79-90 (Mar 2010)

27. Trouilleux, F.: Identification des reprises et interprétation automatique des expressions pronominales dans des textes en français. Ph.D. thesis, Université Blaise Pascal (2001)

28. Vieira, R., Salmon-Alt, S., Schang, E.: Multilingual corpora annotation for processing definite descriptions. In: Proceedings of PorTAL (2002)

29. Witten, I.H., Frank, E., Trigg, L., Hall, M., Holmes, G., Cunningham, S.J.: Weka: Practical machine learning tools and techniques with java implementations (1999)

30. Yang, X., Su, J., Lang, J., Tan, C.L., Liu, T., Li, S.: An entity-mention model for coreference resolution with inductive logic programming. In: Proceedings of ACL'08. pp. 843-851 (2008)

31. Yang, X., Zhou, G., Su, J., Tan, C.L.: Coreference resolution using competition learning approach. In: Proceedings of ACL'03. pp. 176-183 (2003) 